\title{
Aurora kinase inhibitor VX-680 suppresses the proliferation and migration of HUVECs and angiogenesis
}

\author{
XUEJIAO SUN ${ }^{1 *}$, SHISHI NIU ${ }^{1 *}$, ZHEN ZHANG $^{1 *}$, ANYAN WANG $^{1}$, CHENGYUAN YANG $^{1}$, \\ ZICHAN GUO ${ }^{1}$, YUEPENG HAO ${ }^{1}$, XIAOZHONG LI $^{2}$ and XIAOXIA WANG ${ }^{1}$ \\ ${ }^{1}$ Department of Biochemistry and Molecular Biology, Shanxi Medical University, Taiyuan, Shanxi 030001; \\ ${ }^{2}$ Department of Emergency, Shanxi Provincial People's Hospital, Taiyuan, Shanxi 030012, P.R. China
}

Received July 12, 2018; Accepted September 1, 2018

DOI: $10.3892 / \mathrm{mmr} .2019 .9996$

\begin{abstract}
Angiogenesis serves a key role in tumor growth and metastasis. VX-680, a potent inhibitor targeting the Aurora kinase family, is widely used in the inhibition of tumor progression. However, the effect of VX-680 on angiogenesis remains unknown. The present study identified that VX-680 inhibited human umbilical vein endothelial cell (HUVEC) proliferation and promoted HUVEC apoptosis by inducing the cleavage of PARP and caspase-3. VX-680 also markedly decreased the migration and tube formation of HUVECs in a dose-dependent manner. In addition, VX-680 significantly suppressed the formation of blood vessels in a dose-dependent manner confirmed by a chicken embryo chorioallantoic membrane assay in vivo. Furthermore, VX-680 inhibited the expression levels of vascular endothelial growth factor and phosphorylated RAC- $\alpha$ serine/threonine-protein kinase in HUVECs. These results suggested that VX-680 suppressed angiogenesis and may be a potential novel anti-angiogenic agent.
\end{abstract}

\section{Introduction}

Angiogenesis refers to the formation of new capillaries on the existing blood vessels that provide oxygen and nutrients to the various tissues and organs of the body $(1,2)$, which involves a number of steps, including endothelial cell proliferation,

Correspondence to: Dr Xiaoxia Wang, Department of Biochemistry and Molecular Biology, Shanxi Medical University, 56 Xinjian South Road, Taiyuan, Shanxi 030001, P.R. China

E-mail: wxiaoxia99007@126.com

Mr. Xiaozhong Li, Department of Emergency, Shanxi Provincial People's Hospital, 29 Shuangta Street, Taiyuan, Shanxi 030012, P.R. China

E-mail: lixiaozhong@126.com

*Contributed equally

Key words: VX-680, human umbilical vein endothelial cells, angiogenesis, proliferation, migration migration, and endothelial cells forming blood vessels with a tubular structure (3). Even a tumor tissue of only $2-3 \mathrm{~mm}^{3}$ requires a constant blood and nutrient supply (2). Moreover, tumor angiogenesis enables tumor cells to depart from the primary tumor site and enter the circulation, facilitating tumor metastasis (4). Therefore, suppression of tumor angiogenesis has been a promising approach in the prevention of tumor growth and metastasis.

VX-680 is a potent inhibitor that targets the Aurora kinase family (Aurora-A, Aurora-B and Aurora-C) by binding to the ATP site of the kinase domain (5-7). It has been reported that VX-680 is able to inhibit cell proliferation and induce cellular apoptosis in various cancer types, including ovarian cancer, cervical cancer, renal cell carcinoma and acute promyelocytic leukemia (8-12). Moreover, VX-680 has been confirmed to be a promising, specific therapeutic tool for eradicating metastases (13). VX-680 also enhances chemosensitivity to cisplatin and Taxol $(14,15)$. Therefore, VX-680 may represent a novel approach to the treatment of cancer. However, its role in angiogenesis remains unknown.

The present study evaluated the effects of VX-680 on angiogenesis by examining endothelial cell function in vitro and the formation of blood vessels in chicken embryo chorioallantoic membranes (CAMs) in vivo. The results indicated that VX-680 may be used as an anti-angiogenic agent.

\section{Materials and methods}

Regents. VX-680 was purchased from BioVision, Inc.(Milpitas, CA, USA). RPMI-1640 and fetal bovine serum (FBS) were purchased from HyClone (GE Healthcare Life Sciences, Logan, UT, USA). DAPI was purchased from Beijing Solarbio Science \& Technology Co., Ltd. (Beijing, China). Matrigel was purchased from Corning, Inc. (Corning, NY, USA). Mouse anti-poly [ADP-ribose] polymerase (PARP; cat. no. 551024; 1:1,000) antibody was obtained from BD Biosciences (San Jose, CA, USA). Mouse anti-procaspase-3 (cat. no. sc-271759; 1:1,000) antibody, mouse anti-vascular endothelial growth factor A (VEGFA; cat. no. sc-7269; 1:1,000) antibody and mouse anti- $\beta$-actin (cat. no. sc-8432; 1:1,500) antibody were obtained from Santa Cruz Biotechnology, Inc. (Dallas, TX, USA). Rabbit anti-RAC- $\alpha$ serine/threonine-protein kinase (AKT; cat. no. 9272; 1:1,000) antibody and rabbit 
anti-phosphorylated (p)-AKT (cat. no. 9271; 1:1,000) antibody were obtained from Cell Signaling Technology, Inc. (Danvers, MA, USA).

Cell culture. HUVECs (human umbilical vein endothelial cells) were purchased from Jiangyin Qi Biotechnology Co., Ltd. (Hai'an, China) and cultured in RPMI-1640 supplemented with $10 \%$ FBS under standard culture conditions $\left(37^{\circ} \mathrm{C}\right.$ with $5 \% \mathrm{CO}_{2}$ ).

Cell viability assay. Cell viability was assessed by MTT assay. HUVECs $\left(1 \times 10^{4}\right.$ cells/well) were cultured in 96-well plates and treated for 24, 48, 72 and $96 \mathrm{~h}$ with VX-680 (0, 1.5 and $2.25 \mu \mathrm{M})$. Subsequently, $50 \mu \mathrm{l}$ MTT $(5 \mathrm{mg} / \mathrm{ml})$ was added into each plate and incubated for a further $4 \mathrm{~h}$. A volume of $150 \mu \mathrm{l}$ dimethyl sulfoxide was added to solubilize the formazan. The absorbance was measured at $490 \mathrm{~nm}$ using a microplate reader.

Colony formation assay. HUVECs were plated in $60 \mathrm{~mm}$ cell culture dishes $\left(2 \times 10^{3}\right.$ cells/dish) and treated with VX-680 $(0,1.5$ and $2.25 \mu \mathrm{M})$ for $24 \mathrm{~h}$. The medium was refreshed, and the cells were cultured for another 14 days. HUVECs were stained with $0.1 \%$ crystal violet for $15 \mathrm{~min}$ at room temperature after fixing with $70 \%$ methanol for $10 \mathrm{~min}$ at room temperature. The colonies were photographed and manually counted using a Universal Hood II Molecular Imager Gel System (Bio-Rad Laboratories, Inc., Hercules, CA, USA) and an inverted microscope (magnification, $\mathrm{x} 40$ ).

Apoptosis assay. HUVECs $\left(2 \times 10^{5}\right.$ cells/well) were seeded in $60 \mathrm{~mm}$ culture dishes and incubated with VX-680 $(0,1.5$ and $2.25 \mu \mathrm{M}$ ) for $24 \mathrm{~h}$. The cells were fixed with methanol for $20 \mathrm{~min}$ at room temperature and stained with $0.1 \%$ DAPI for $15 \mathrm{~min}$ at room temperature. The cells were photographed using a fluorescence microscope (magnification, x200).

Western blot analysis. HUVECs were cultured in $60 \mathrm{~mm}$ cell culture dishes and treated with VX-680 $(0,1.5$ and $2.25 \mu \mathrm{M})$ for $24 \mathrm{~h}$, and the total protein was extracted using Radioimmunoprecipitation Assay lysis buffer (cat. no. P0013B; Beyotime Institute of Biotechnology, Haimen, China). The proteins in the cell lysates were quantified using the Bradford method. The cell lysates were mixed with sample buffer and boiled for $5 \mathrm{~min}$. The proteins ( $80 \mu \mathrm{g} / \mathrm{lane})$ were separated by $10 \%$ SDS-PAGE and subsequently transferred to nitrocellulose (NC) membranes. The $\mathrm{NC}$ membranes were blocked with 5\% skimmed milk at room temperature for $60 \mathrm{~min}$. The membranes were subsequently incubated with the aforementioned primary antibodies overnight at $4^{\circ} \mathrm{C}$, followed by incubation with horseradish-peroxidase-conjugated secondary antibodies [cat. nos. W401B and W402B; 1:3,000; Promega (Beijing) Biotech Co., Ltd. Beijing, China] for $60 \mathrm{~min}$ at room temperature. The protein bands were observed using enhanced chemiluminescence reagent (cat. no. 29050; Engreen Biosystem Co., Ltd., Beijing, China) and the band intensities were measured using ImageJ software (version 1.48; National Institutes of Health, Bethesda, MD, USA).

Wound healing assay. HUVECs were incubated to complete confluence, scratched using pipette tips and washed three
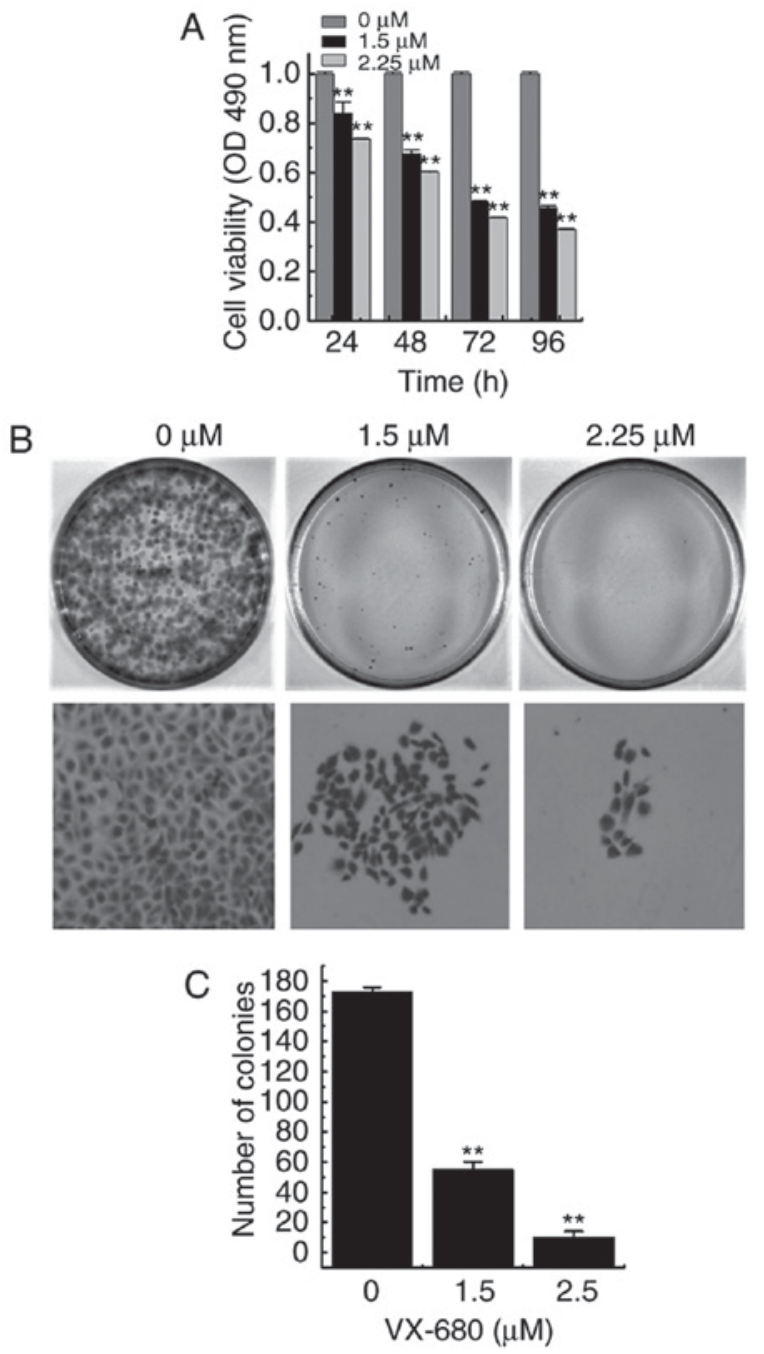

Figure 1. VX-680 reduces HUVEC viability. (A) The MTT assay demonstrated that VX-680 inhibited HUVEC growth in a dose- and time-dependent manner. (B) Representative images of colony formation are displayed (lower panel: magnification, $x 40)$. (C) The quantification of the colony formation assay is presented. Data are presented as the mean \pm standard deviation of three independent experiments. ${ }^{* *} \mathrm{P}<0.01$ vs. respective control group. HUVEC, human umbilical vein endothelial cell; OD, optical density.

times with PBS. HUVECs were treated with VX-680 $(0,1.5$ and $2.25 \mu \mathrm{M})$ for $24 \mathrm{~h}$, and images were captured under a light microscope (magnification, x100) at 0 and $24 \mathrm{~h}$. Cell migration ability was analyzed using Image-Pro Plus (version 6.0; Media Cybernetics, Rockville, MD, USA).

Tube formation assay. Matrigel (50 $\mu 1 /$ well) was added to 96-well plates and incubated at $37^{\circ} \mathrm{C}$ for $40 \mathrm{~min}$. HUVECs were treated with VX-680 $(0,1.5$ and $2.25 \mu \mathrm{M})$ for $24 \mathrm{~h}$. The cell density was adjusted to $2.5 \times 10^{4}$ cells/well, and the cells were cultured in pre-coated 96-well plates for $3 \mathrm{~h}$. HUVECs were photographed using an inverted microscope (magnification, $x 40$ ). Results were quantified by measuring the tube length.

Chicken embryo CAM assay. A $1 \mathrm{~cm}$ diameter window was opened in the shell of each egg $(n=9)$ containing 7-day-old chicken embryo to expose the chicken embryo CAM. Disinfected filter papers were added to the CAM, which 
A

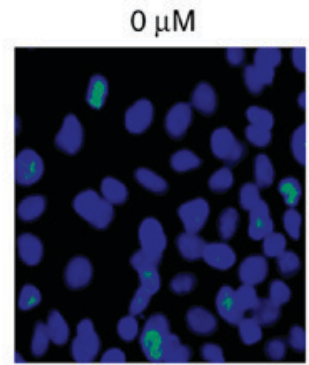

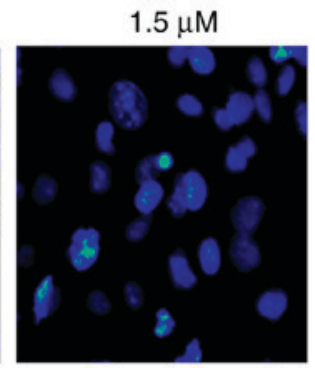
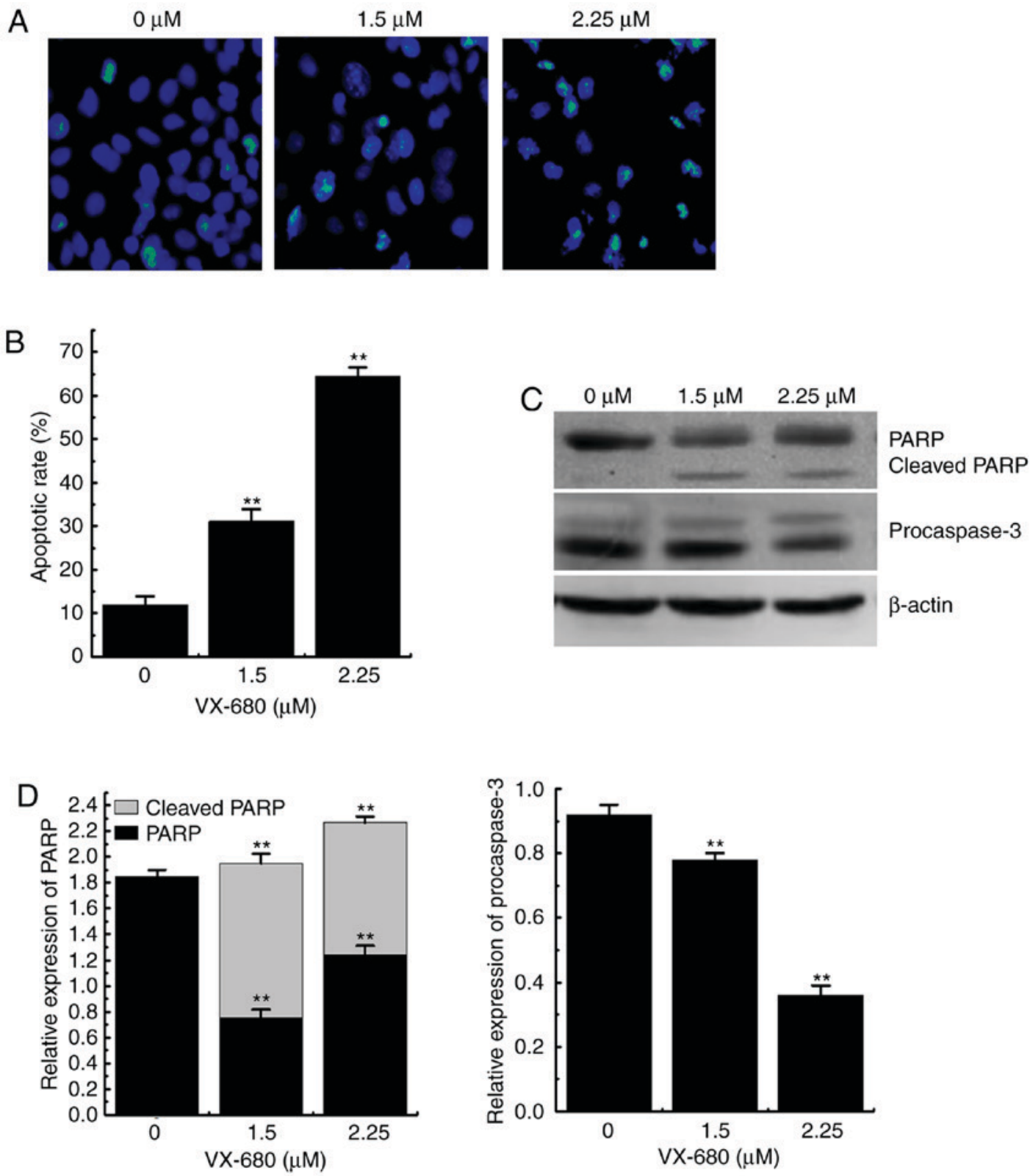

Figure 2. VX-680 induces HUVEC apoptosis. DAPI staining was used to analyze the effect of VX-680 on the apoptosis of HUVECs. (A) Representative images (magnification, x200) and (B) quantification are presented. (C) The expression levels of PARP and procaspase-3 were assessed by western blot analysis and (D) quantified. $\beta$-actin was used as the internal control. Data are presented as the mean \pm standard deviation of three replicates. ${ }^{* *} \mathrm{P}<0.01$ vs. respective control group. HUVEC, human umbilical vein endothelial cell; PARP, poly [adenosine diphosphate-ribose] polymerase.

were steeped in VX-680 $(0,1.5$ and $2.25 \mu \mathrm{M})$. After $48 \mathrm{~h}$, chicken embryos were fixed for 15 min with stationary solution (methanol: acetone 1:1) at room temperature. CAMs were harvested and images were captured using a Universal Hood II Molecular Imager Gel System (Bio-Rad Laboratories, Inc.). The number of blood vessels was manually counted in each image.

Statistical analysis. Data were analyzed statistically using SPSS 16.0 software (SPSS, Inc., Chicago, IL, USA). The results are expressed as the mean \pm standard deviation from three independent experiments. The differences between groups were assessed by one-way analysis of variance followed by Dunnett's multiple comparison post hoc test. $\mathrm{P}<0.05$ was considered to indicate a statistically significant difference.

\section{Results}

VX-680 reduces HUVEC viability. The present study examined the effect of VX-680 on HUVEC proliferation by MTT analysis. As presented in Fig. 1A, VX-680 significantly inhibited HUVEC viability in a dose- and time-dependent manner. To further assess the anti-proliferative ability of VX-680 on HUVECs, a colony formation assay was performed. As presented in Fig. 1B and C, VX-680 reduced colony size and number in comparison with control cells. The data indicated that VX-680 suppresses HUVEC viability.

VX-680 induces HUVEC apoptosis. To determine whether apoptosis contributes to the growth inhibition mediated by VX-680, morphological alterations in the cell nuclei 
A

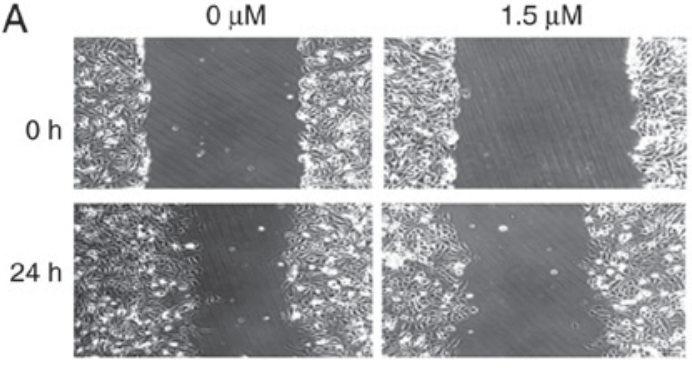

$2.25 \mu \mathrm{M}$

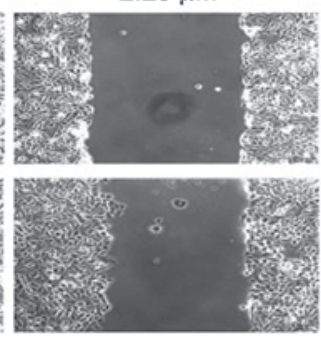

B

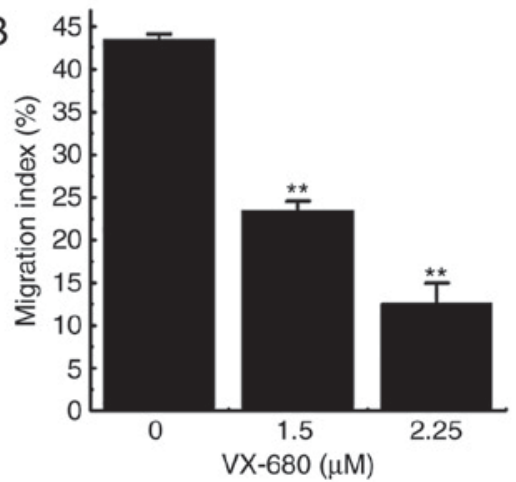

Figure 3. VX-680 attenuates the migratory ability of human umbilical vein endothelial cells. (A) Representative images of the wound healing assay are presented (magnification, x100). (B) The quantification of the wound healing assay is presented. Data are presented from three independent experiments (mean \pm standard deviation). ${ }^{* *} \mathrm{P}<0.01$ vs. control group.

were examined by DAPI staining in HUVECs. The results revealed that HUVECs exhibited nuclear fragmentation and condensation following treatment with VX-680, which are morphological features of apoptosis (Fig. 2A). The rates of apoptosis in VX-680-treated cells (31.11 and 64.32\%) were higher compared with those in control cells (11.94\%; Fig. 2B). Consistent with the results of the DAPI staining, VX-680 resulted in evident PARP cleavage, whereas PARP cleavage was weaker in control cells. Furthermore, the procaspase-3 in VX-680-treated cells was significantly decreased compared with that observed in control cells (Fig. 2C and D), indicating cleavage of caspase-3 in cells following VX-680 treatment, although the cleaved fragment of caspase-3 was not detected. The results suggested that VX-680 promoted the apoptosis of HUVECs by inducing the cleavage of PARP and caspase-3.

VX-680 inhibits HUVEC migration. Endothelial cell migration is a critical step in angiogenesis $(3,4)$. To examine whether VX-680 regulated the migratory characteristics of endothelial cells, a wound healing assay was performed. As hypothesized, the results demonstrated that a significant reduction in the migration of HUVECs was observed when cells were treated with VX-680 compared with the control cells (Fig. 3). The results demonstrated that VX-680 significantly inhibited the migratory ability of HUVECs.

VX-680 suppresses angiogenesis in vitro and in vivo. To evaluate the anti-angiogenic ability of VX-680, a HUVEC tube formation assay was conducted in vitro in addition to a chicken embryo CAM assay in vivo. As presented in Fig. 4A, HUVECs were seeded on Matrigel and formed robust capillary-like structures in the control group after $3 \mathrm{~h}$. By contrast, the tube formation on Matrigel of HUVECs pre-treated with VX-680 for $24 \mathrm{~h}$ was decreased. Approximately 13.39 and $65.4 \%$ destruction of the tube network was observed when HUVECs were treated with VX-680 at 1.5 and $2.25 \mu \mathrm{M}$, respectively (Fig. 4B). Similarly, the control group exhibited formation of new capillaries and branches in the CAM, whereas VX-680 inhibited angiogenesis. Quantitative analysis revealed that 1.5 and $2.25 \mu \mathrm{M}$ VX-680 caused a 1.5- and 2.5-fold reduction in the number of blood vessels, respectively (Fig. 4C and D). These findings suggested that VX-680 suppressed angiogenesis in vitro and in vivo.
VX-680 downregulates VEGFA expression and the AKT signaling pathway. VEGFA, as a crucial angiogenic factor, promotes the proliferation and migration of endothelial cells and stimulates the differentiation of endothelial cells into capillaries $(16,17)$. Therefore, the present study investigated the effects of VX-680 on VEGFA protein expression by western blot analysis. The data demonstrated that VX-680 significantly decreased the expression of VEGFA in a concentration-dependent manner compared with the control (Fig. 5A). In addition, AKT serves a crucial role in regulating a series of endothelial cell functions, including cell proliferation and migration $(3,18)$. The results demonstrated that VX-680 led to a decrease in p-AKT expression in a dose-dependent manner. However, the total expression of AKT was almost unaffected (Fig. 5B). These data suggested that the suppression of angiogenesis by VX-680 may be mediated in part by the downregulation of VEGFA expression and AKT signaling in HUVECs; further studies are required to test this hypothesis.

\section{Discussion}

The majority of cancer mortality is due to tumor metastasis, and angiogenesis serves a pivotal role in tumor proliferation and metastasis (16-20). Thus, there is an urgent need to develop effective anti-angiogenic agents for the treatment of cancer. The Aurora serine/threonine kinase family, including Aurora kinases $\mathrm{A}, \mathrm{B}$, and $\mathrm{C}$, is critical in regulating mitotic processes. A number of studies have demonstrated that aberrant Aurora kinase activity and expression have been observed in numerous human cancer types (21-23). Therefore, Aurora kinase family members may serve as potential molecular targets of anti-tumor therapy. VX-680, as an Aurora kinase inhibitor, is able to inhibit cell proliferation and metastasis, and induce apoptosis in tumor cells by blocking the ATP-binding site of the serine/threonine kinase $(8-15,24)$. However, the potential effect of VX-680 on angiogenesis remains poorly understood.

Endothelial cell proliferation and apoptosis serve critical roles in the process of angiogenesis $(4,16,17)$. In the present study, it was observed that VX-680 effectively inhibited HUVEC proliferation in a time- and dose-dependent manner. DAPI staining and analysis of the cleavage of PARP and caspase- 3 indicated that VX-680 promoted HUVEC apoptosis. Endothelial cell migration also serves a key role in 
A

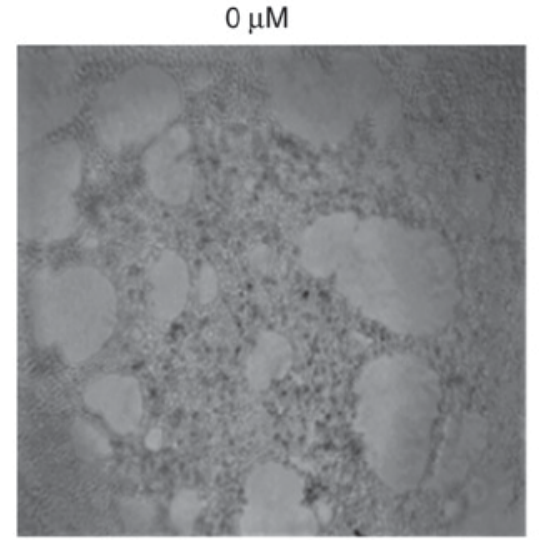

B

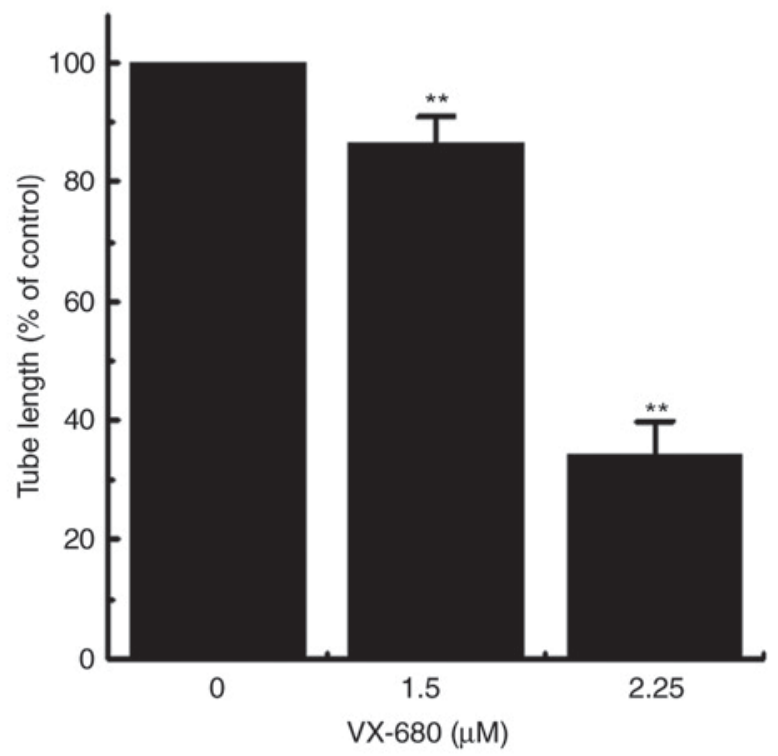

D

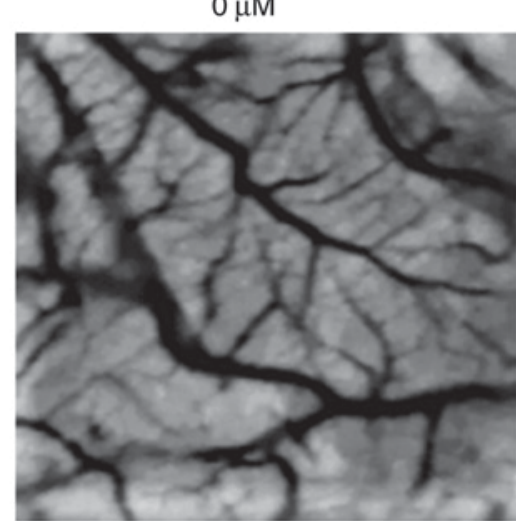

$1.5 \mu \mathrm{M}$

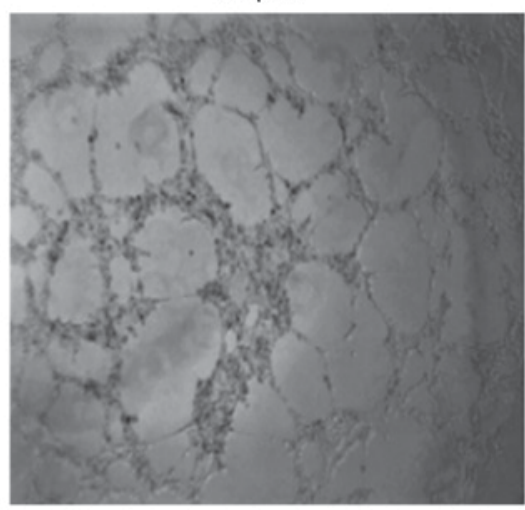

C

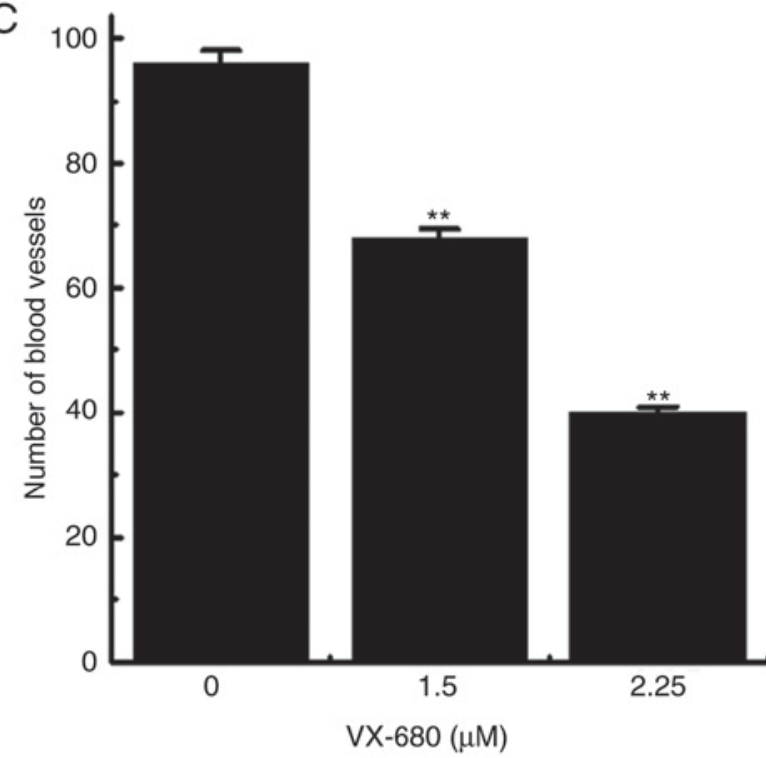

$1.5 \mu \mathrm{M}$

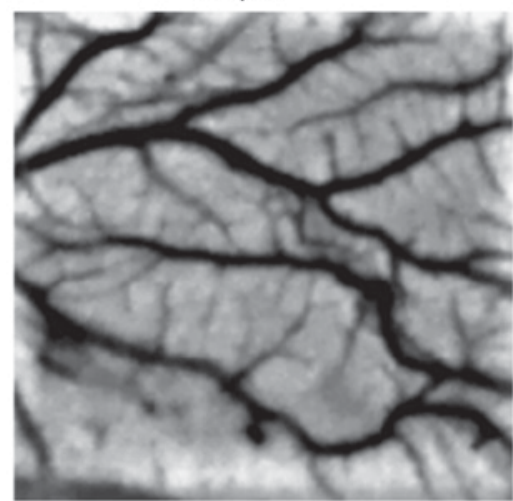

$2.25 \mu \mathrm{M}$

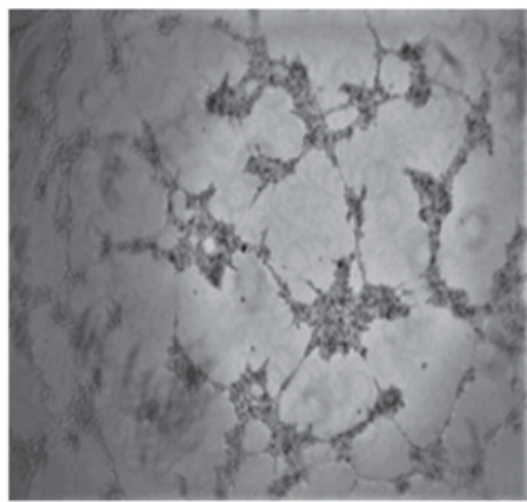

VX-680 $(\mu \mathrm{M})$

Figure 4. VX-680 inhibits angiogenesis in vitro and in vivo. (A) Representative images (magnification, $\mathrm{x} 40$ ) and (B) quantification of human umbilical vein endothelial cell tube formation cultured on Matrigel-coated plates with VX-680. The inhibitory effects of VX-680 on angiogenesis were detected by chicken embryo CAM assay in vivo. (C) Quantification and (D) representative images of the chicken embryo CAM assay following treatment with VX-680, captured using the Universal Hood II Molecular Imager Gel System. Data are presented from three independent experiments (mean \pm standard deviation). ${ }^{* *} \mathrm{P}<0.01 \mathrm{vs}$. respective control group. CAM, chorioallantoic membrane.

angiogenesis $(3,4)$. According to the wound healing assay, it was demonstrated that VX-680 significantly inhibited the migratory ability of HUVECs. In addition, a tube formation assay was performed to analyze the anti-angiogenic effect of VX-680, which is a well-known angiogenesis experiment in vitro (25). The results indicated that VX-680 significantly reduced the formation of capillary-like structures. Furthermore, the chicken embryo CAM assay in vivo was used to test the anti-angiogenic effect of VX-680. The results displayed that VX-680 significantly inhibited the formation of blood vessels in a dose-dependent manner. These results illustrated that VX-680 inhibited angiogenesis in vitro and in vivo. 

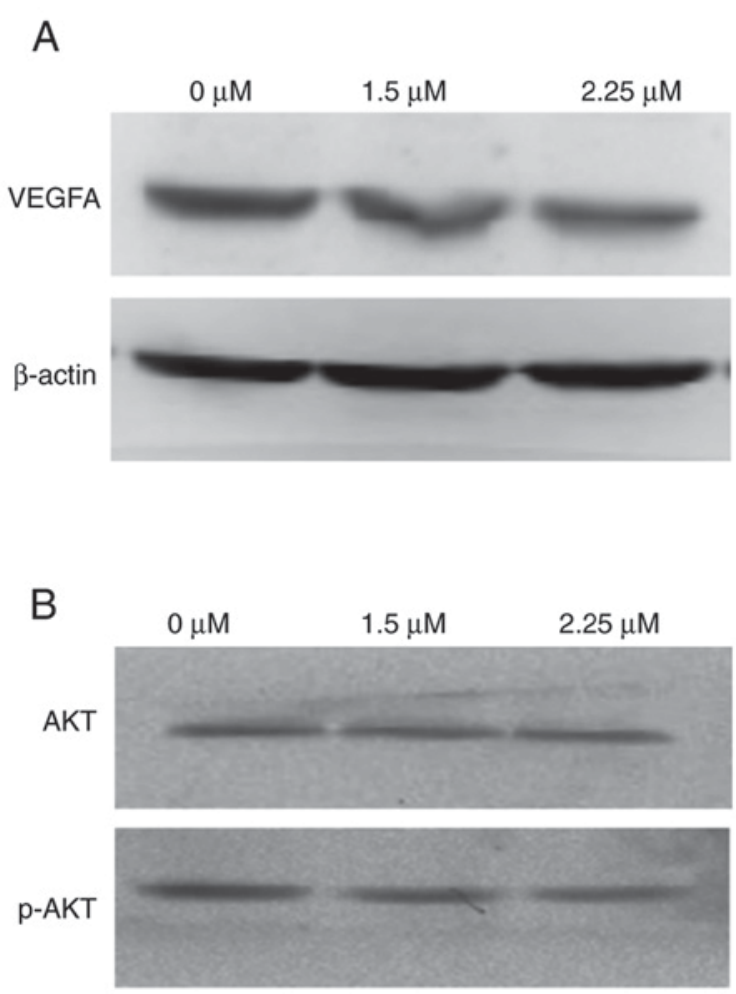

$\beta$-actin

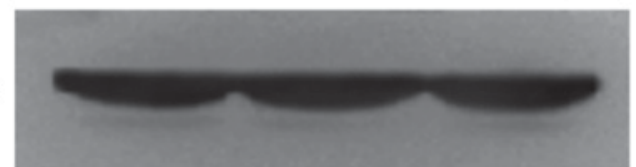

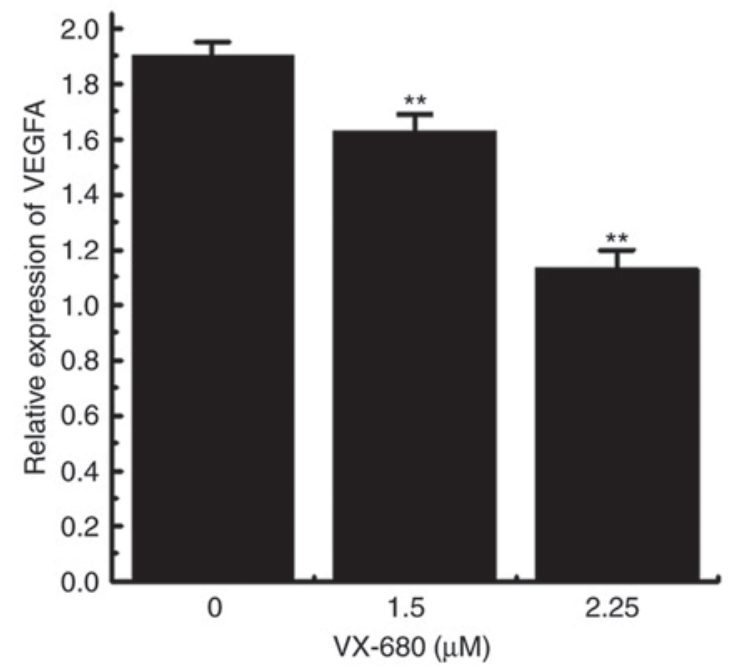

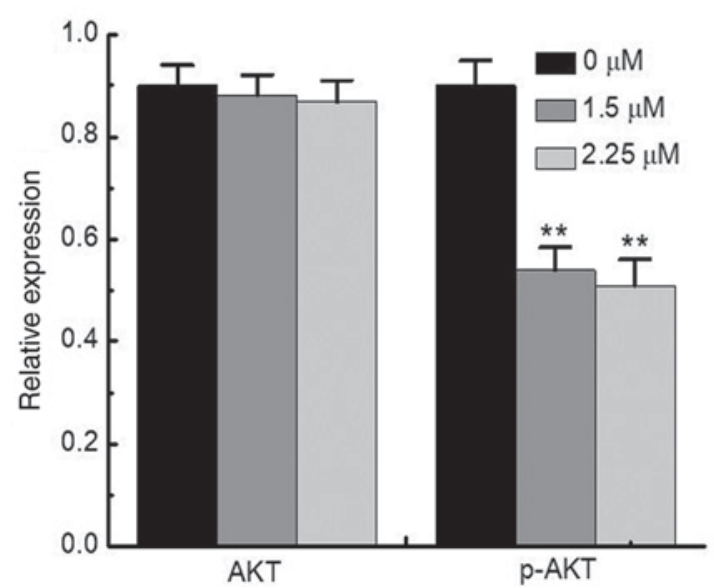

Figure 5. VX-680 decreases the expression of VEGFA and p-AKT. The expression levels of (A) VEGFA, and (B) AKT and p-AKT in human umbilical vein endothelial cells treated with VX-680 were analyzed by western blotting. $\beta$-actin was used as the internal control. Data are presented as the mean \pm standard deviation of three replicates. ${ }^{* *} \mathrm{P}<0.01$ vs. respective control group. VEGFA, vascular endothelial growth factor A; AKT, RAC- $\alpha$ serine/threonine-protein kinase; p, phosphorylated.

The role of VEGFA in angiogenesis is essential. VEGFA is overexpressed in the majority of types of cancer and is involved in most of the stages of tumor angiogenesis $(16,17,26)$. Studies have demonstrated that anti-angiogenic drugs principally target VEGFA and the associated signaling pathways $(4,16,17,27)$. The present results demonstrated that VX-680 significantly decreased the expression of VEGFA. AKT is a type of serine/threonine protein kinase and serves an important role in cell proliferation and migration $(27,28)$. The activation of the AKT signaling pathway can stimulate angiogenesis $(18,20)$. The present study identified that the phosphorylation of AKT was inhibited by VX-680 while the total expression of AKT was unaltered. The above results further demonstrated that VX-680 serves an anti-angiogenic role.

In conclusion, the results of the present study indicated that VX-680 exerted effective anti-angiogenic activity, and VX-680 may be a new anti-angiogenic drug for cancer therapy.

\section{Acknowledgements}

Not applicable.

\section{Funding}

The present study was supported by grants from the National Natural Science Foundation of China (grant no. 81372676) and Natural Science Foundation of Shanxi Province (grant no. 201601D011130).

\section{Availability of data and materials}

All data generated or analyzed during this study are included in this published article.

\section{Authors' contributions}

$\mathrm{XS}, \mathrm{SN}$ and ZZ performed the experiments, and analyzed and interpreted the data. AW, CY, ZG and YH were involved in collecting the data. XS, XL and XW designed the study and were responsible for writing the manuscript. All authors read and approved the manuscript.

\section{Ethics approval and consent to participate}

Not applicable. 


\section{Patient consent for publication}

Not applicable.

\section{Competing interests}

The authors declare that they have no competing interests.

\section{References}

1. Kubota Y: Tumor angiogenesis and anti-angiogenic therapy. Keio J Med 61: 47-56, 2012.

2. Abdelrahim M, Konduri S, Basha R, Philip PA and Baker CH: Angiogenesis: An update and potential drug approaches. Int J Oncol 36: 5-18, 2010.

3. Su M, Huang J, Li J, Qin X, Tang X, Jin F, Chen S, Jiang C, Zou Z, Peng K, et al: ZLM-7 exhibits anti-angiogenic effects via impaired endothelial cell function and blockade of VEGF/VEGFR-2 signaling. Oncotarget 7: 19018-19030, 2016.

4. Saraswati S, Kanaujia PK, Kumar S, Kumar R and Alhaider AA Tylophorine, a phenanthraindolizidine alkaloid isolated from Tylophora indica exerts antiangiogenic and antitumor activity by targeting vascular endothelial growth factor receptor 2-mediated angiogenesis. Mol Cancer 12: 82, 2013.

5. Fiskus W, Wang Y, Joshi R, Rao R, Yang Y, Chen J, Kolhe R, Balusu R, Eaton K, Lee P, et al: Cotreatment with vorinostat enhances activity of MK-0457 (VX-680) against acute and chronic myelogenous leukemia cells. Clin Cancer Res 14: 6106-6115, 2008.

6. Doggrell SA: Dawn of Aurora kinase inhibitors as anticancer drugs. Expert Opin Investig Drugs 13: 1199-1201, 2004

7. Cheetham GM, Charlton PA, Golec JM and Pollard JR: Structural basis for potent inhibition of the Aurora kinases and a T315I multi-drug resistant mutant form of Abl kinase by VX-680 Cancer Lett 251: 323-329, 2007.

8. Lin YG, Immaneni A, Merritt WM, Mangala LS, Kim SW, Shahzad MM, Tsang YT, Armaiz-Pena GN, Lu C, Kamat AA, et al: Targeting aurora kinase with MK-0457 inhibits ovarian cancer growth. Clin Cancer Res 14: 5437-5446, 2008.

9. Jin X, Mo Q, Zhang Y, Gao Y, Wu Y, Li J, Hao X, Ma D, Gao Q and Chen P: The p38 MAPK inhibitor BIRB796 enhances the antitumor effects of VX680 in cervical cancer. Cancer Biol Ther 17: 566-576, 2016.

10. Li Y, Zhang ZF, Chen J, Huang D, Ding Y, Tan MH, Qian CN, Resau JH, Kim H and The BT: VX680/MK-0457, a potent and selective Aurora kinase inhibitor, targets both tumor and endothelial cells in clear cell renal cell carcinoma. Am J Transl Res 2: 296-308, 2010

11. Xu DR, Huang S, Long ZJ, Chen JJ, Zou ZZ, Li J, Lin DJ and Liu Q: Inhibition of mitotic kinase Aurora suppresses Akt-1 activation and induces apoptotic cell death in all-trans retinoid acid-resistant acute promyelocytic leukemia cells. J Transl Med 9: 74, 2011.

12. Giles FJ, Swords RT, Nagler A, Hochhaus A, Ottmann OG, Rizzieri DA, Talpaz M, Clark J, Watson P, Xiao A, et al: MK-0457, an Aurora kinase and BCR-ABL inhibitor, is active in patients with BCR-ABL T315I leukemia. Leukemia 27: 113-117, 2013.

13. Pezzani R, Rubin B, Bertazza L, Redaelli M, Barollo S, Monticelli H, Baldini E, Mian C, Mucignat C, Scaroni C, et al: The aurora kinase inhibitor VX-680 shows anti-cancer effects in primary metastatic cells and the SW13 cell line. Invest New Drugs 34: 531-540, 2016.
14. Sun JM, Yang LN, Xu H Chang B, Wang HY and Yang G: Inhibition of Aurora A promotes chemosensitivity via inducing cell cycle arrest and apoptosis in cervical cancer cells. Am J Cancer Res 5: 1133-1145, 2015.

15. Yao R, Zheng J, Zheng W, Gong Y, Liu W and Xing R: VX680 suppresses the growth of HepG2 cells and enhances the chemosensitivity to cisplatin. Oncol Lett 7: 121-124, 2014.

16. Bertolini F, Marighetti P, Martin-Padura I, Mancuso P, Hu-Lowe DD, Shaked Y and D'Onofrio A: Anti-VEGF and beyond: shaping a new generation of anti-angiogenic therapies for cancer. Drug Discov Today 16: 1052-1060, 2011.

17. Roskoski R Jr: Vascular endothelial growth factor (VEGF) signaling in tumor progression. Crit Rev Oncol Hematol 62: 179-213, 2007.

18. Cui HJ, Yang AL, Zhou HJ, Wang C, Luo JK, Lin Y, Zong YX and Tang T: Buyang huanwu decoction promotes angiogenesis via vascular endothelial growth factor receptor-2 activation through the PI3K/Akt pathway in a mouse model of intracerebral hemorrhage. BMC Complement Altern Med 15: 91, 2015.

19. Wu XY, Xu H, Wu ZF, Chen C, Liu JY, Wu GN, Yao XQ, Liu FK, Li G and Shen L: Formononetin, a novel FGFR2 inhibitor, potently inhibits angiogenesis and tumor growth in preclinical models. Oncotarget 6: 44563-44578, 2015.

20. Sharma S, Guru SK, Manda S, Kumar A Mintoo MJ, Prasad VD, Sharma PR, Mondhe DM, Bharate SB and Bhushan S: A marine sponge alkaloid derivative 4-chloro fascaplysin inhibits tumor growth and VEGF mediated angiogenesis by disrupting PI3K/Akt/mTOR signaling cascade. Chem Biol Interact 275: 47-60, 2017.

21. Yan M, Wang C, He B, Yang M, Tong M, Long Z, Liu B, Peng F, $\mathrm{Xu}$ L, Zhang Y, et al: Aurora-A Kinase: A potent oncogene and target for cancer therapy. Med Res Rev 36: 1036-1079, 2016.

22. D'Assoro AB, Haddad T and Galanis E: Aurora-A kinase as a promising therapeutic target in cancer. Front Oncol 5: 295, 2016.

23. Portella G, Passaro C and Chieffi P: Aurora B: A new prognostic marker and therapeutic target in cancer. Curr Med Chem 18: 482-96, 2011.

24. Seymour JF, Kim DW, Rubin E, Haregewoin A, Clark J, Watson P, Hughes T, Dufva I, Jimenez JL, Mahon FX, et al: A phase 2 study of MK-0457 in patients with BCR-ABL T315I mutant chronic myelogenous leukemia and philadelphia chromosome-positive acute lymphoblastic leukemia. Blood Cancer J 4: e238, 2014.

25. Ponce ML: Tube formation: An in vitro matrigel angiogenesis assay. Methods Mol Biol 467: 183-188, 2009.

26. Kim ES, Serur A, Huang J, Manley CA, McCrudden KW, Frischer JS, Soffer SZ, Ring L, New T, Zabski S, et al: Potent VEGF blockade causes regression of coopted vessels in a model of neuroblastoma. Proc Natl Acad Sci USA 99: 11399-11404, 2002.

27. Saraswati S, Kumar S and Alhaider AA: $\alpha$-santalol inhibits the angiogenesis and growth of human prostate tumor growth by targeting vascular endothelial growth factor receptor 2-mediated AKT/mTOR/P70S6K signaling pathway. Mol Cancer 12: 147, 2013.

28. Du J, Wang R, Yin L, Fu Y, Cai Y, Zhang Z and Liang A: BmK CT enhances the sensitivity of temozolomide-induced apoptosis of malignant glioma U251 cells in vitro through blocking the AKT signaling pathway. Oncol Lett 15: 1537-1544, 2018. 